\title{
A PRIORI AND A POSTERIORI ANALYSIS OF THE QUASINONLOCAL QUASICONTINUUM METHOD IN 1D
}

\author{
CHRISTOPH ORTNER
}

\begin{abstract}
For a next-nearest neighbour pair interaction model in a periodic domain, a priori and a posteriori analyses of the quasinonlocal quasicontinuum method (QNL-QC) are presented. The results are valid for large deformations and essentially guarantee a one-to-one correspondence between atomistic solutions and QNL-QC solutions. The analysis is based on consistency error estimates in negative norms, novel a priori and a posteriori stability estimates, and a quantitative inverse function theorem.
\end{abstract}

\section{INTRODUCTION}

Quasicontinuum (QC) methods [10, 16, 17, 19, 26, 27] are a class of prototypical schemes for coupling atomistic models of solids to continuum elasticity models. They combine, in principle, the accuracy of atomistic descriptions of defects with the efficiency of continuum finite element descriptions of long-range elastic fields. For details on the historical development of the QC method see [16, 17.

Not all QC methods are equally reliable in modelling large atomistic systems. Recent analyses of QC methods [3, 5, 6, 8, 9, 15, 18, have identified various sources of inconsistencies (in the sense of numerical analysis; see also Section 3) and instabilities due to inadequate treatment of the interface. Three methods that have emerged as the most promising candidates are: (i) the force-based QC method [4. 26]; (ii) the quasinonlocal QC (QNL-QC) method [27] and its generalization 10]; and (iii) a new variant of the QNL-QC method for finite range pair interactions that is consistent for general interface geometries [25].

All contributions to the analysis of QC methods cited above consider only linearized problems, except for [18] where small deformations from a uniform reference state are admitted. The main purpose of the present paper is to introduce an analytical framework that allows a generalization of these results to genuinely nonlinear situations. To present the new ideas that are required to achieve this in the simplest possible setting, we will focus on a one-dimensional model problem where the atomistic model is formulated in terms of a second neighbour pair interaction energy with applied dead loads. For this model, the QNL-QC method of Shimokawa et al. [27] was previously analyzed in [5, 18. However, the techniques used there

Received by the editor November 7, 2009 and, in revised form, May 15, 2010.

2010 Mathematics Subject Classification. Primary 65N12, 65N15, 70C20.

Key words and phrases. Quasicontinuum method, quasinonlocal coupling, finite deformations, sharp stability, error analysis.

This work was supported by the EPSRC Critical Mass Programme "New Frontiers in the Mathematics of Solids".

(C)2010 American Mathematical Society Reverts to public domain 28 years from publication 
would be difficult to extend to the large deformation regime, for which a new approach will be presented here, based on consistency error estimates in negative Sobolev norms and sharp a priori and a posteriori stability estimates. Moreover, care will be taken throughout this paper that the techniques can, in principle, be extended to higher dimensions and to situations with defects. This will primarily be achieved by modifying ideas from 21] (where a Galerkin projection without continuum approximation is analyzed), so that no higher regularity results for the Hessian operator of the atomistic or QC energy functionals are required.

The present paper also develops the first residual-based a posteriori error analysis for the QNL-QC method. Theorem 5.3 presents an a posteriori error estimate for the QNL-QC method that, at the same time, establishes the existence of an exact solution to which the estimate applies. The application of goal oriented a posteriori error control to QC methods was pioneered in [1, 2, 24].

Throughout the text interesting and challenging open problems are discussed.

Finally, it should be remarked that the purpose of this paper is to present a new framework for the analysis of the QC method in the simplest possible setting that still allows the main features one observes in applications: nonsmooth solutions and large deformations. Numerical experiments illustrating the results, as well as further interesting observations will be presented after introducing finite element coarse graining into the framework 22 .

Outline. We begin, in Section 2.1, by presenting an atomistic model problem in a periodic formulation that avoids the difficulties posed by boundaries. In Section 2.2. we formulate the QNL-QC approximation without coarse graining. For an analysis of the QNL-QC including finite element coarse graining of the continuum region see 22. In the remainder of Section 2 we discuss some features of the atomistic model and introduce the necessary technical background for the subsequent analysis.

In Section 3 we discuss the concepts of truncation error and residual in the context of the QC method. Previous work [5] has analyzed the truncation error in weighted $\ell^{p}$-norms, which leads to suboptimal truncation error estimates. As a result, somewhat subtle and technically demanding ideas were required to still obtain (quasi-)optimal error estimates. By contrast, we will see here that if the "correct" norm is chosen, namely a negative Sobolev norm, then these complications can be completely avoided. In [18, an interesting variant of a negative norm was used that is useful for the nonlinear analysis but does not lead to (quasi-)optimal error estimates (see Remarks 3.4 and 5.2 ).

The subject of Section 4 is a careful and general stability analysis of the QNL-QC method. First, an idea from [6] will be extended to obtain a priori stability results for large deformations. The main novel contribution in this section, however, is the $a$ posteriori stability result in Theorem 4.7. This section concludes with a brief discussion of the difficulties encountered when defects are present, and how the results might be generalized.

Finally, in Section 5 we combine the consistency and stability analyses of the previous sections to obtain a priori as well as a posteriori existence results and error estimates. The techniques used here are not very different from [18, however, the point of view taken is a very different one and leads to a different set of existence results. Moreover, the sharper consistency and stability results of the present paper lead to error estimates that are truly (quasi-)optimal in the atomistic spacing and the smoothness of the atomistic solution. See Remarks 5.2 and 5.4 for more detail. 


\section{Atomistic model and QNL-QC approximation}

2.1. An atomistic model problem. In atomistic models surfaces create boundary layers in the deformation field and must therefore be considered defects in the lattice. We avoid this issue, by working in an infinite lattice $\varepsilon \mathbb{Z}$, where $\varepsilon>0$ is the reference lattice spacing. Since the functional analysis becomes unnecessarily difficult in this infinite domain we will, moreover, restrict the set of admissible deformations to those which are $N$-periodic displacements from the reference lattice, that is, we define

$$
\mathscr{Y}=F x+\mathscr{U},
$$

where $x_{\xi}=\varepsilon \xi$ for $\xi \in \mathbb{Z}, F>0$ is a macroscopic deformation gradient, and where

$$
\mathscr{U}=\left\{u \in \mathbb{R}^{\mathbb{Z}}: u_{\xi+N}=u_{\xi} \quad \forall \xi \in \mathbb{Z}, \text { and } \sum_{\xi=1}^{N} u_{\xi}=0\right\} .
$$

The condition $\sum_{\xi=1}^{N} u_{\xi}=0$ is an artefact of the periodic boundary conditions, and ensures locally unique solvabililty of the equilibrium equations to the models that are introduced below. We set $\varepsilon=1 / N$ throughout so that the reference period has unit length.

We denote the algebraic dual of $\mathscr{U}$ by $\mathscr{U}^{*}$. Equipped with the weighted $\ell^{2}$-inner product

$$
\langle v, w\rangle=\varepsilon \sum_{\xi=1}^{N} v_{\xi} w_{\xi},
$$

$\mathscr{U}$ is a Hilbert space, and hence, any $T \in \mathscr{U}^{*}$ can be represented by a function $z_{T} \in \mathscr{U}$ via $T[v]=\left\langle z_{T}, v\right\rangle$ for $v \in \mathscr{U}$. Thus, even though forces are usually best understood as elements of $\mathscr{U}^{*}$, we will always identify them with "functions" $f \in \mathscr{U}$.

The stored energy per period of a deformation $y \in \mathscr{Y}$ is given by a next-nearest neighbour pair interaction model,

$$
\Phi_{\mathrm{a}}(y):=\varepsilon \sum_{\xi=1}^{N}\left(\phi\left(y_{\xi}^{\prime}\right)+\phi\left(y_{\xi}^{\prime}+y_{\xi+1}^{\prime}\right)\right),
$$

where $v_{\xi}^{\prime}=\varepsilon^{-1}\left(v_{\xi}-v_{\xi-1}\right)$ for $v \in \mathbb{R}^{\mathbb{Z}}$, and $\phi$ is a Lennard-Jones type interaction potential satisfying the following properties:

$(\phi 1) \quad \phi \in \mathrm{C}^{3}((0,+\infty] ; \mathbb{R})$, and

$(\phi 2) \quad$ there exists $r_{*}>0$ s.t. $\phi$ is convex in $\left(0, r_{*}\right)$ and concave in $\left(r_{*},+\infty\right)$.

By $\phi \in \mathrm{C}^{3}((0,+\infty] ; \mathbb{R})$ we mean that $\phi$ and its first three derivatives are bounded in any interval $(\delta,+\infty), \delta>0$. Implicitly, we actually assume that $\phi(r)$ and its derivatives decay rapidly to zero as $r \rightarrow \infty$. This justifies the next-nearest neighbour interaction model.

We assume, for simplicity, that the atomistic system is subjected to a dead load $f \in \mathscr{U}$, so that the total energy of a deformation $y \in \mathscr{Y}$ is given by

$$
\Phi_{\mathrm{a}}^{\mathrm{tot}}(y)=\Phi_{\mathrm{a}}(y)-\langle f, y\rangle .
$$

Our goal is to solve the minimization problem

$$
y_{\mathrm{a}} \in \operatorname{argmin} \Phi_{\mathrm{a}}^{\mathrm{tot}}(\mathscr{Y}),
$$


where argmin denotes the set of local minimizers. The first order necessary optimality condition (or, Euler-Lagrange equation) associated with (2.2) is

$$
D \Phi_{\mathrm{a}}^{\mathrm{tot}}\left(y_{\mathrm{a}}\right)[u]=0 \quad \forall u \in \mathscr{U} .
$$

A deformation $y_{\mathrm{a}} \in \mathscr{Y}$ satisfying (2.3) is called an equilibrium (or critical point). If an equilibrium $y_{\mathrm{a}}$ also satisfies the second order sufficient optimality condition,

$$
D^{2} \Phi_{\mathrm{a}}^{\mathrm{tot}}\left(y_{\mathrm{a}}\right)[u, u]>0 \quad \forall u \in \mathscr{U} \backslash\{0\},
$$

then we say that $y$ is a strongly stable equilibrium. A strongly stable equilibrium is an isolated local minimizer of $\Phi_{\mathrm{a}}^{\text {tot }}$ in $\mathscr{Y}$.

2.2. The quasinonlocal QC method. If a deformation $y \in \mathscr{Y}$ is smooth, in the sense that $y_{\xi}^{\prime} \approx y_{\xi+1}^{\prime}$ for all $\xi$, then we can approximate the second-neighbour terms by

$$
\phi\left(y_{\xi}^{\prime}+y_{\xi+1}^{\prime}\right) \approx \frac{1}{2}\left\{\phi\left(2 y_{\xi}^{\prime}\right)+\phi\left(2 y_{\xi+1}^{\prime}\right)\right\},
$$

which leads to an approximate stored energy functional

$$
\Phi_{\mathrm{cb}}(y)=\varepsilon \sum_{\xi=1}^{N}\left\{\phi\left(y_{\xi}^{\prime}\right)+\phi\left(2 y_{\xi}^{\prime}\right)\right\}=\varepsilon \sum_{\xi=1}^{N} \phi_{\mathrm{cb}}\left(y_{\xi}^{\prime}\right),
$$

where $\phi_{\mathrm{cb}}(r)=\phi(r)+\phi(2 r)$ is called the Cauchy-Born stored energy function. The Cauchy-Born stored energy functional $\Phi_{\mathrm{cb}}$ is local and its minimization can be achieved efficiently by means of finite element methodology. (Note, though, that in 1D this is not really an issue [21].) Moreover, it is considered an excellent approximation to the atomistic model in a smooth deformation regime (see [12] for a formal asymptotic theory of the small deformation regime).

If a minimizer of the atomistic model, which we are trying to compute, has defects in a small localized region but is smooth elsewhere, then we should couple the atomistic model to the continuum Cauchy-Born model. The QNL-QC method 27] is the simplest energy-based coupling method that is consistent for next-nearest neighbour models (see Section 3 for a discussion of consistency). For the simple pair interaction energy that we use in this paper it can be defined as follows.

Let $\mathcal{A} \subset\{1, \ldots, N\}$ be the atomistic region and let $\mathcal{C}=\{1, \ldots, N\} \backslash \mathcal{A}$ be the continuum region. The nearest neigbour terms are left unchanged, while, for $\xi \in \mathcal{C}$, the next-nearest neighbour interaction $\phi\left(y_{\xi}^{\prime}+y_{\xi+1}^{\prime}\right)$ is approximated as in (2.5). This procedure leads to the QNL-QC stored energy functional

$$
\Phi_{\mathrm{qc}}(y)=\varepsilon \sum_{\xi=1}^{N} \phi\left(y_{\xi}^{\prime}\right)+\varepsilon \sum_{\xi \in \mathcal{A}} \phi\left(y_{\xi}^{\prime}+y_{\xi+1}^{\prime}\right)+\varepsilon \sum_{\xi \in \mathcal{C}} \frac{1}{2}\left\{\phi\left(2 y_{\xi}^{\prime}\right)+\phi\left(2 y_{\xi+1}^{\prime}\right)\right\} .
$$

Upon defining the total energy

$$
\Phi_{\mathrm{qc}}^{\mathrm{tot}}(y)=\Phi_{\mathrm{qc}}(y)-\langle f, y\rangle,
$$

in the QNL-QC method, we solve the minimization problem

$$
y_{\mathrm{qc}} \in \operatorname{argmin} \Phi_{\mathrm{qc}}^{\mathrm{tot}}(\mathscr{Y}) \text {. }
$$

The associated first order optimality condition in variational form is

$$
D \Phi_{\mathrm{qc}}^{\mathrm{tot}}\left(y_{\mathrm{qc}}\right)[u]=0 \quad \forall u \in \mathscr{U} .
$$




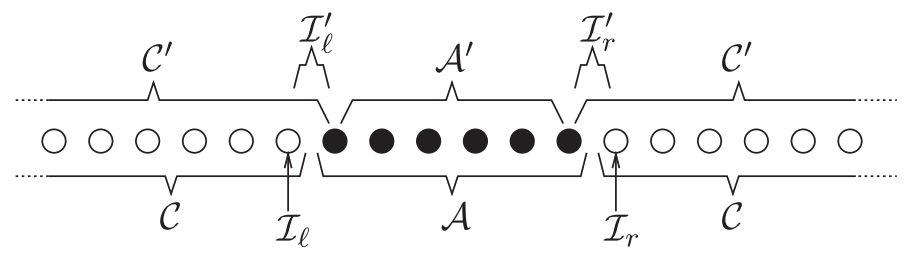

Figure 1. Visualization of the atomistic region, the continuum region, the interface sets, as well as their counterparts for bounds, defined in Section 2.2 ,

Remark 2.1. For practical reasons, it is important that this approximation can be rewritten in the form

$$
\Phi_{\mathrm{qc}}(y)=\Phi_{\mathrm{qc}}^{\mathcal{A}}(y)+\Phi_{\mathrm{qc}}^{\mathcal{I}}(y)+\Phi_{\mathrm{qc}}^{\mathcal{C}}(y),
$$

where $\Phi_{\mathrm{qc}}^{\mathcal{A}}, \Phi_{\mathrm{qc}}^{\mathcal{I}}$, and $\Phi_{\mathrm{qc}}^{\mathcal{C}}$ are the contributions from the atomistic region, the interfacial region, and the continuum region, respectively, and so that $\Phi_{\mathrm{qc}}^{\mathcal{A}}$ is precisely the atomistic model restricted to (a subset of) $\mathcal{A}$, that $\Phi_{\mathrm{qc}}^{\mathcal{C}}$ is precisely the CauchyBorn approximation restricted to (a subset of) $\mathcal{C}$, and $\Phi_{\mathrm{qc}}^{\mathcal{I}}$ is an interface correction that is inexpensive to compute. This formulation allows for finite element coarsening of the continuum region. For more details on this splitting and its importance, see 22. For the purposes of the analysis presented in this paper, however, (2.6) is the most useful formulation.

For future reference, we define the left- and right-interface sets (left and right are understood in relation to the atomistic region(s)) as follows:

$$
\mathcal{I}_{\ell}=\left\{\xi \in \mathcal{C}: \xi+1 \in \mathcal{A}_{\#}\right\} \quad \text { and } \quad \mathcal{I}_{r}=\left\{\xi \in \mathcal{C}: \xi-1 \in \mathcal{A}_{\#}\right\}
$$

where $\mathcal{A}_{\#}=\bigcup_{j \in \mathbb{Z}}(N j+\mathcal{A})$ is a periodically extended atomistic region. The union $\mathcal{I}=\mathcal{I}_{\ell} \cup \mathcal{I}_{r}$ is simply called the interface. A visualization of these and the following definitions is provided in Figure 1 .

We assume throughout that

$$
\mathcal{I}_{\ell} \cap \mathcal{I}_{r}=\emptyset .
$$

This means, that any connected component of the continuum region must contain at least two atoms.

In addition, since we will often write expressions in terms of bonds rather than atoms, it is sometimes more convenient to use the following variants of these sets:

$$
\mathcal{I}_{\ell}^{\prime}=\mathcal{I}_{\ell}+1, \quad \mathcal{I}_{r}^{\prime}=\mathcal{I}_{r}, \quad \mathcal{A}^{\prime}=\mathcal{A} \backslash \mathcal{I}_{\ell}^{\prime}, \quad \mathcal{C}^{\prime}=\mathcal{C} \cup \mathcal{I}_{\ell}^{\prime}, \quad \text { and } \quad \mathcal{I}^{\prime}=\mathcal{I}_{r}^{\prime} \cup \mathcal{I}_{\ell}^{\prime} .
$$

These definitions are made mostly for the sake of an intuitive notation in the main results. On a first reading, it may be best to ignore these subtle differences and simply bear in mind that the sets $\mathcal{A}^{\prime}$, etc., are variants of the originals that allow for an attractive presentation.

2.3. A bound on next-nearest neighbour interactions. It will be a crucial ingredient in the stability analysis of the QNL-QC method to assume that nextnearest neighbour interactions remain in the concave region of the interaction potential, that is, we assume that the solutions of the atomistic model and of the 
QNL-QC method satisfy

$$
y_{\xi}^{\prime} \geq r_{*} / 2 \quad \forall \xi \in\{1, \ldots, N\} .
$$

For most interesting interaction potentials, the case $y_{\xi}^{\prime}<r_{*} / 2$ can only be achieved under extreme compressive forces that will not usually be observed in experiments. As a matter of fact, one can safely assume that under such extreme conditions a classical potential (as opposed to a potential based on quantum mechanics) and zero temperature statics constitute an inappropriate model to begin with.

Note, moreover, that the condition $y_{\xi}^{\prime} \geq r_{*} / 2$ also ensures that $\phi$ and its derivatives satisfy uniform bounds. For future reference, we define the constants

$$
C_{j}(s):=\sup _{r \geq s} \phi^{(j)}(r) \quad \text { for } s>0 \text { and for } k=0,1, \ldots,
$$

where $\phi^{(j)}$ denotes the $j$ th derivative of $\phi$.

2.4. Further notation and an auxiliary result. The second and third finite differences are defined, for $v \in \mathbb{R}^{\mathbb{Z}}$, by

$$
\begin{aligned}
v_{\xi}^{\prime \prime} & :=\varepsilon^{-2}\left(v_{\xi+1}-2 v_{\xi}+v_{\xi-1}\right), \quad \text { and } \\
v_{\xi}^{\prime \prime \prime} & :=\varepsilon^{-3}\left(v_{\xi+1}-3 v_{\xi}+3 v_{\xi-1}-v_{\xi-2}\right) .
\end{aligned}
$$

For $v \in \mathscr{U}$, the functions $v^{\prime}, v^{\prime \prime}$, and $v^{\prime \prime \prime}$ are understood as $N$-periodic functions. We normally associate the function value $v_{\xi}$ and the second difference $v_{\xi}^{\prime \prime}$ with the atom $\xi$, while the first difference $v_{\xi}^{\prime}$ and the third difference $v_{\xi}^{\prime \prime \prime}$ are normally associated with the cell, or bond, $(\xi-1, \xi)$.

In addition to the weighted $\ell^{2}$-inner product $\langle\cdot, \cdot\rangle$ we define the weighted $\ell^{p}$-norms

$$
\|v\|_{\ell_{\varepsilon}^{p}}:=\left\{\begin{array}{cl}
\left(\varepsilon \sum_{\xi=1}^{N}\left|v_{\xi}\right|^{p}\right)^{1 / p}, & 1 \leq p<\infty \\
\max _{\xi=1, \ldots, N}\left|v_{\xi}\right|, & p=\infty .
\end{array}\right.
$$

We normally drop the subscript in $\ell_{\varepsilon}^{\infty}$ and write $\ell^{\infty}$ instead.

The space $\mathscr{U}$ can also be equipped with the Sobolev-like norms

$$
\|v\|_{\mathscr{U}^{1, p}}=\left\|v^{\prime}\right\|_{\ell_{\varepsilon}^{p}} \quad \text { for } v \in \mathscr{U} \quad \text { and } p \in[1, \infty],
$$

and is then denoted $\mathscr{U}^{1, p}$. For $p^{\prime}=p /(p-1)$, the norm on the topological dual $\mathscr{U}^{-1, p}:=\left(\mathscr{U}^{1, p^{\prime}}\right)^{*}$ of $\mathscr{U}^{1, p^{\prime}}$ is

$$
\|T\|_{\mathscr{U}^{-1, p}}:=\|T\|_{\left(\mathscr{U}^{1, p^{\prime}}\right)^{*}}=\sup _{\substack{v \in \mathscr{U}^{\prime} \\\|v\|_{\mathscr{U}^{1, p^{\prime}}}=1}} T[v], \quad \text { for } T \in \mathscr{U}^{-1, p} .
$$

If $\Psi: \mathscr{Y} \rightarrow \mathbb{R}$ is (Fréchet) differentiable, then its first variation is denoted $D \Psi$ and is understood as a nonlinear map from $\mathscr{Y}$ to $\mathscr{U}^{*}$, that is, for $y \in \mathscr{Y}$, $D \Psi(y) \in \mathscr{U}^{*}, v \mapsto D \Psi(y)[v]$. Similarly, the second variation $D^{2} \Psi$ is understood as a nonlinear map from $\mathscr{Y}$ to $L\left(\mathscr{U}, \mathscr{U}^{*}\right)$, that is, for $y \in \mathscr{Y}, D^{2} \Psi(y) \in L\left(\mathscr{U}, \mathscr{U}^{*}\right)$. Equivalently, $D^{2} \Psi(y)$ can be understood as a symmetric bilinear form on $\mathscr{U} \times \mathscr{U}$, $(v, w) \mapsto D^{2} \Psi(y)[v, w]$. We will henceforth make no distinction between these different interpretations and use whichever is most convenient at any given moment.

Lemma 2.2 (Inverse Function Theorem). Let $X, Y$ be Banach spaces, $A$ an open subset of $X$, and let $\mathscr{F}: A \rightarrow Y$ be Fréchet differentiable. Suppose that $x_{0} \in A$ 
satisfies the conditions

$$
\begin{aligned}
& \left\|\mathscr{F}\left(x_{0}\right)\right\|_{Y} \leq \eta, \quad\left\|D \mathscr{F}\left(x_{0}\right)^{-1}\right\|_{L(Y, X)} \leq \sigma, \\
& \overline{B_{X}\left(x_{0}, 2 \eta \sigma\right)} \subset A, \\
& \left\|D \mathscr{F}\left(x_{1}\right)-D \mathscr{F}\left(x_{2}\right)\right\|_{L(X, Y)} \leq L\left\|x_{1}-x_{2}\right\|_{X} \quad \text { for } \quad\left\|x_{j}-x_{0}\right\|_{X} \leq 2 \eta \sigma, \\
& \text { and } 2 L \sigma^{2} \eta<1,
\end{aligned}
$$

then there exists a unique $x \in X$ such that $\mathscr{F}(x)=0$ and $\left\|x-x_{0}\right\|_{X} \leq 2 \eta \sigma$.

Proof. The result follows, for example, by applying Theorem 2.1 in 20 with the choices $R=2 \eta \sigma, \omega\left(x_{0}, R\right)=L R$ and $\bar{\omega}\left(x_{0}, R\right)=\frac{1}{2} L R^{2}$. Similar results can be obtained by tracking the constants in most proofs of the inverse function theorem, and assuming local Lipschitz continuity of $D \mathscr{F}$.

\section{Consistency}

It was pointed out in [5] that the QNL-QC method is not consistent. This observation was based on the fact that the authors computed the consistency error in the $\ell^{\infty}$-norm and considered the limit as $\varepsilon \rightarrow 0$. As a matter of fact, the QNLQC is consistent in appropriate negative norms (see also [18, Lemma 5.11], and 22 for a more general discussion). However, before we prove this result, we briefly review the notions of truncation error and residual, and explain more clearly what we mean by consistency.

Let $y_{\mathrm{a}} \in \operatorname{argmin} \Phi_{\mathrm{a}}^{\text {tot }}(\mathscr{Y})$, then the truncation error (associated with this solution) is the linear functional $T \in \mathscr{U}^{*}$, which measures the extent to which $y_{\text {a }}$ fails to satisfy (2.8):

$$
T[u]=D \Phi_{\mathrm{qc}}^{\mathrm{tot}}\left(y_{\mathrm{a}}\right)[u]=D \Phi_{\mathrm{qc}}\left(y_{\mathrm{a}}\right)[u]-\langle f, u\rangle .
$$

Conversely, if $y_{\mathrm{qc}} \in \operatorname{argmin} \Phi_{\mathrm{qc}}^{\mathrm{tot}}(\mathscr{Y})$, then the linear functional $R \in \mathscr{U}^{*}$ that measures the extent to which $y_{\mathrm{qc}}$ fails to satisfy (2.3) is called the residual (of the approximate solution $\left.y_{\mathrm{qc}}\right)$ :

$$
R[u]=D \Phi_{\mathrm{a}}^{\mathrm{tot}}\left(y_{\mathrm{qc}}\right)[u]=D \Phi_{\mathrm{a}}\left(y_{\mathrm{qc}}\right)[u]-\langle f, u\rangle .
$$

Since $y_{\mathrm{a}}$ satisfies (2.3) and $y_{\mathrm{qc}}$ satisfies (2.8) we deduce that

$$
T=D \Phi_{\mathrm{qc}}\left(y_{\mathrm{a}}\right)-D \Phi_{\mathrm{a}}\left(y_{\mathrm{a}}\right), \text { and } R=D \Phi_{\mathrm{a}}\left(y_{\mathrm{qc}}\right)-D \Phi_{\mathrm{qc}}\left(y_{\mathrm{qc}}\right) .
$$

Thus, estimating the truncation error will automatically give us a residual estimate and vice-versa. Hence, we will call both $R$ and $T$ simply the consistency error. (This equivalence is only partially valid if coarse-graining is taken into account.)

Traditionally, we call a numerical method consistent if the truncation error tends to zero, in a suitable sense, as the mesh size, or another discretization parameter, tends to zero. In the present case, since $\varepsilon$ is fixed (it is best to think of $\varepsilon$ to be of the order $O\left(10^{-3}\right)$ or $O\left(10^{-4}\right)$ ), we cannot make such a definition. We will therefore use the term rather loosely and simply discuss the order of consistency of the method. For example, first order consistency would mean that the consistency error can be bounded by $\varepsilon$ and a factor that depends on the smoothness of $y$. However, even this nomenclature is not wholly appropriate as the QNL-QC method has different orders of consistency in different parts of the domain. This is demonstrated in the following theorem, which provides a sharp bound on the consistency error of the QNL-QC method in the $\mathscr{U}^{-1, p}$-norms, $1 \leq p \leq \infty$. Even though we will later only 
use $\mathscr{U}^{-1,2}$ estimates, the more general case is included here since it requires no additional work.

Theorem 3.1 (Consistency in $\mathscr{U}^{-1, p}$ ). Let $y \in \mathscr{Y}$ such that $\min _{\xi \in \mathbb{Z}} y_{\xi}^{\prime}>0$, then

$$
\left\|D \Phi_{a}(y)-D \Phi_{q c}(y)\right\|_{\mathscr{U}^{-1, p}} \leq \varepsilon \bar{C}_{2}\left\|y^{\prime \prime}\right\|_{\ell_{\varepsilon}^{p}(\mathcal{I})}+\varepsilon^{2} \bar{C}_{3}\left(\left\|y^{\prime \prime \prime}\right\|_{\ell^{p}\left(\mathcal{C}^{\prime} \backslash \mathcal{I}^{\prime}\right)}+\left\|y^{\prime \prime}\right\|_{\ell_{\varepsilon}^{2 p}(\mathcal{C})}^{2}\right),
$$

where $\bar{C}_{i}=C_{i}\left(2 \min _{\xi \in \mathcal{C}^{\prime}} y_{\xi}^{\prime}\right), i=2,3$.

Proof. The first variations of the atomistic and QNL-QC functionals at $y$ are, respectively, given by

$$
D \Phi_{\mathrm{a}}(y)[u]=\varepsilon \sum_{\xi=1}^{N} \phi^{\prime}\left(y_{\xi}^{\prime}\right) u_{\xi}^{\prime}+\varepsilon \sum_{\xi=1}^{N} \phi^{\prime}\left(y_{\xi}^{\prime}+y_{\xi+1}^{\prime}\right)\left[u_{\xi}^{\prime}+u_{\xi+1}^{\prime}\right]
$$

and

$$
\begin{aligned}
D \Phi_{\mathrm{qc}}(y)[u]=\varepsilon \sum_{\xi=1}^{N} \phi^{\prime}\left(y_{\xi}^{\prime}\right) u_{\xi}^{\prime} & +\varepsilon \sum_{\xi \in \mathcal{A}} \phi^{\prime}\left(y_{\xi}^{\prime}+y_{\xi+1}^{\prime}\right)\left[u_{\xi}^{\prime}+u_{\xi+1}^{\prime}\right] \\
& +\varepsilon \sum_{\xi \in \mathcal{C}} \frac{1}{2}\left\{2 \phi^{\prime}\left(2 y_{\xi}^{\prime}\right) u_{\xi}^{\prime}+2 \phi^{\prime}\left(2 y_{\xi+1}^{\prime}\right) u_{\xi+1}^{\prime}\right\} .
\end{aligned}
$$

The consistency error $T:=D \Phi_{\mathrm{a}}(y)-D \Phi_{\mathrm{qc}}(y)$ therefore satisfies

$$
T[u]=\varepsilon \sum_{\xi \in \mathcal{C}}\left\{\phi^{\prime}\left(y_{\xi}^{\prime}+y_{\xi+1}^{\prime}\right)\left[u_{\xi}^{\prime}+u_{\xi+1}^{\prime}\right]-\phi^{\prime}\left(2 y_{\xi}^{\prime}\right) u_{\xi}^{\prime}-\phi^{\prime}\left(2 y_{\xi+1}^{\prime}\right) u_{\xi+1}^{\prime}\right\},
$$

for $u \in \mathscr{U}$. From (3.3) we can immediately obtain a first-order consistency error estimate, however, one can improve upon this by taking into account symmetries of the interaction law.

If we collect all coefficients that premultiply a term $u_{\xi}^{\prime}$, then we obtain secondorder errors in the "interior" of the continuum region and first-order errors in the interface. To see this, we recall the definitions of the interface regions from Section 2.2. and compute

$$
\begin{aligned}
T[u]=\varepsilon & \sum_{\xi \in \mathcal{I}_{\ell}}\left\{\phi^{\prime}\left(y_{\xi}^{\prime}+y_{\xi+1}^{\prime}\right)-\phi^{\prime}\left(2 y_{\xi+1}^{\prime}\right)\right\} u_{\xi+1}^{\prime} \\
& +\varepsilon \sum_{\xi \in \mathcal{I}_{r}}\left\{\phi^{\prime}\left(y_{\xi}^{\prime}+y_{\xi+1}^{\prime}\right)-\phi^{\prime}\left(2 y_{\xi}^{\prime}\right)\right\} u_{\xi}^{\prime} \\
& +\varepsilon \sum_{\xi \in \mathcal{C}^{\prime} \backslash \mathcal{I}^{\prime}}\left\{\phi^{\prime}\left(y_{\xi-1}^{\prime}+y_{\xi}^{\prime}\right)+\phi^{\prime}\left(y_{\xi}^{\prime}+y_{\xi+1}^{\prime}\right)-2 \phi^{\prime}\left(2 y_{\xi}^{\prime}\right)\right\} u_{\xi}^{\prime} .
\end{aligned}
$$

Taylor expansions of the first and second terms in curled brackets yield

$$
\begin{aligned}
\phi^{\prime}\left(y_{\xi}^{\prime}+y_{\xi+1}^{\prime}\right)-\phi^{\prime}\left(2 y_{\xi+1}^{\prime}\right) & =-\varepsilon \phi^{\prime \prime}\left(2 y_{\xi+1}^{\prime}\right) y_{\xi}^{\prime \prime}+\frac{1}{2} \varepsilon^{2} \phi^{\prime \prime \prime}\left(\vartheta_{1, \xi}\right)\left|y_{\xi}^{\prime \prime}\right|^{2}, \quad \text { and } \\
\phi^{\prime}\left(y_{\xi}^{\prime}+y_{\xi+1}^{\prime}\right)-\phi^{\prime}\left(2 y_{\xi}^{\prime}\right) & =\varepsilon \phi^{\prime \prime}\left(2 y_{\xi}^{\prime}\right) y_{\xi}^{\prime \prime}+\frac{1}{2} \varepsilon^{2} \phi^{\prime \prime \prime}\left(\vartheta_{2, \xi}\right)\left|y_{\xi}^{\prime \prime}\right|^{2},
\end{aligned}
$$

where $\vartheta_{1, \xi} \in \operatorname{conv}\left\{y_{\xi}^{\prime}+y_{\xi+1}^{\prime}, 2 y_{\xi+1}^{\prime}\right\}$ and $\vartheta_{2, \xi} \in \operatorname{conv}\left\{y_{\xi}^{\prime}+y_{\xi+1}^{\prime}, 2 y_{\xi}^{\prime}\right\}$, and hence $\left|\phi^{\prime \prime \prime}\left(\vartheta_{i, \xi}\right)\right| \leq C_{3}\left(2 \min _{\xi \in \mathcal{C}^{\prime}} y_{\xi}^{\prime}\right)=: \bar{C}_{3}$ for $i=1,2$. Here, conv $A$ denotes the convex hull of a set $A \subset \mathbb{R}$. 
To expand the third term in curled brackets in (3.4), we use (3.5) twice to obtain

$$
\begin{aligned}
\phi^{\prime}\left(y_{\xi}^{\prime}+y_{\xi+1}^{\prime}\right)+\phi^{\prime}\left(y_{\xi-1}^{\prime}+y_{\xi}^{\prime}\right)-2 \phi^{\prime}\left(2 y_{\xi}^{\prime}\right) \\
\quad=\varepsilon^{2} \phi^{\prime \prime \prime}\left(2 y_{\xi}^{\prime}\right) y_{\xi}^{\prime \prime \prime}+\varepsilon^{2} \frac{1}{2}\left\{\phi^{\prime \prime \prime}\left(\vartheta_{2, \xi}\right)\left|y_{\xi}^{\prime \prime}\right|^{2}+\phi^{\prime \prime \prime}\left(\vartheta_{1, \xi-1}\right)\left|y_{\xi-1}^{\prime \prime}\right|^{2}\right\} .
\end{aligned}
$$

Inserting these expansions and bounds into (3.4), and applying several weighted Hölder inqualities we can estimate

$$
\begin{aligned}
|T[u]| \leq & \varepsilon \sum_{\xi \in \mathcal{I}_{\ell}}\left\{\varepsilon \bar{C}_{2}\left|y_{\xi}^{\prime \prime}\right|+\varepsilon^{2} \frac{1}{2} \bar{C}_{3}\left|y_{\xi}^{\prime \prime}\right|^{2}\right\}\left|u_{\xi+1}^{\prime}\right|+\varepsilon \sum_{\xi \in \mathcal{I}_{r}}\left\{\varepsilon \bar{C}_{2}\left|y_{\xi}^{\prime \prime}\right|+\varepsilon^{2} \frac{1}{2} \bar{C}_{3}\left|y_{\xi}^{\prime \prime}\right|^{2}\right\}\left|u_{\xi}^{\prime}\right| \\
& +\varepsilon \sum_{\xi \in \mathcal{C} \backslash \mathcal{I}_{r}}\left\{\varepsilon^{2} \bar{C}_{3}\left|y_{\xi}^{\prime \prime \prime}\right|+\varepsilon^{2} \frac{1}{2} \bar{C}_{3}\left(\left|y_{\xi}^{\prime \prime}\right|^{2}+\left|y_{\xi-1}^{\prime \prime}\right|^{2}\right)\right\}\left|u_{\xi}^{\prime}\right| \\
\leq & \left\{\varepsilon \bar{C}_{2}\left\|y^{\prime \prime}\right\|_{\ell_{\varepsilon}^{p}(\mathcal{I})}+\varepsilon^{2} \bar{C}_{3}\left\|y^{\prime \prime \prime}\right\|_{\ell_{\varepsilon}^{p}\left(\mathcal{C} \backslash \mathcal{I}_{r}\right)}+\varepsilon^{2} \bar{C}_{3}\left\|y^{\prime \prime}\right\|_{\ell_{\varepsilon}^{2 p}(\mathcal{C})}^{2}\right\}\left\|u^{\prime}\right\|_{\ell_{\varepsilon}^{p^{\prime}}} .
\end{aligned}
$$

Remark 3.2. The consistency error has no contribution from the atomistic region, which is not surprising since the model is exact in $\mathcal{A}$. In the continuum region, the consistency error reflects the second-order accuracy of the Cauchy-Born approximation for simple lattices 12. In the interface, the consistency error is only of first order. By estimating $\left\|y^{\prime \prime}\right\|_{\ell_{\varepsilon}^{p}(\mathcal{I})}$ above by $\left\|y^{\prime \prime}\right\|_{\ell^{\infty}(\mathcal{I})}$, we can, however, obtain additional powers of $\varepsilon$,

$$
\varepsilon \bar{C}_{2}\left\|y^{\prime \prime}\right\|_{\ell_{\varepsilon}^{p}(\mathcal{I})} \leq \varepsilon^{1+1 / p} \bar{C}_{2}(\# \mathcal{I})^{1 / p}\left\|y^{\prime \prime}\right\|_{\ell^{\infty}(\mathcal{I})},
$$

but at the cost of a dependency on the size of the interfacial region. Moreover, it should be stressed that such an estimate may hide the reduced accuracy of the QNL-QC method in the interface.

This loss of accuracy at the interface can potentially become problematic if very high accuracy is sought. For example, a P2 finite element discretization of the continuum region will not be able to increase the accuracy of the computation beyond the error introduced in this interface. Thus, a higher order coupling mechanism would be a highly desirable goal.

A finer analysis of this reduced accuracy for a linearized model problem can be found in [5].

Remark 3.3. Generalizations of Theorem 3.1 for a variety of QC methods can be found in [22. There, we also include finite element coarse graining in the analysis. We use negative norm consistency error estimates, similar to Theorem 3.1 .

Remark 3.4. In [18, Lemma 5.11] a negative-norm consistency error estimate with respect to a so-called Spijker norm can be found, which, in the language of the present work, reads

$$
\left|D \Phi_{\mathrm{a}}(y)[u]-D \Phi_{\mathrm{qc}}(y)[u]\right| \leq C \varepsilon\left(\varepsilon^{1 / 2}\left\|u^{\prime}\right\|_{\ell_{\varepsilon}^{2}}\right) .
$$

This estimate follows immediately from (3.6). The reason for choosing this norm is that it guarantees uniform closeness of gradients (i.e., $\varepsilon^{-1 / 2}\left\|w^{\prime}\right\|_{\ell_{\varepsilon}^{2}} \geq\left\|w^{\prime}\right\|_{\ell \infty}$, where $\varepsilon^{-1 / 2}\left\|w^{\prime}\right\|_{\ell_{\varepsilon}^{2}}$ is dual to $\varepsilon^{1 / 2}\left\|u^{\prime}\right\|_{\ell_{\varepsilon}^{2}}$, a fact that is highly useful in the nonlinear analysis. Unfortunately, it leads to suboptimal error estimates. In the analysis of Section 5 , we will circumvent this problem by using inverse estimates, but still retain the optimal truncation error estimates. 


\section{StabiLity}

Having established a fairly sharp consistency error estimate, we turn to the question of stability of the QNL-QC method. Stability estimates are usually easiest to establish in a Hilbert space, particularly when the operator under investigation is coercive. Coercivity of the QNL-QC Hessian evaluated at the reference state $y=F x$ was established in [5, 18, and it was shown in [6] that the estimate in [5] sharply reflects the stability of the full atomistic model. In the following sections we will generalize these results to situations that admit large deformations.

In one dimension, it is relatively straightforward to derive stability bounds in the space $\mathscr{U}^{1, p}$. For example, several stability results for atomistic and quasicontinuum models in the space $\mathscr{U}^{1, \infty}$, which are particularly useful for a nonlinear analysis, were derived in [21]. However, since such results would be very difficult (if not impossible) to obtain in $2 \mathrm{D} / 3 \mathrm{D}$, we will attempt in the next sections to use only $\mathscr{U}^{1,2}$-coercivity estimates in our analysis and still obtain similarly strong nonlinear results.

4.1. Preliminary remarks. Linear stability for minimization problems is normally associated with the coercivity constant (or smallest eigenvalue) in an appropriate function space. Thus, to relate the stability of the atomistic model and that of the QNL-QC method, we would like to prove a result of the type

$$
c_{\mathrm{a}}(y):=\inf _{\substack{u \in \mathscr{U} \\\|u\|_{\mathscr{U}^{1,2}}=1}} D^{2} \Phi_{\mathrm{a}}(y)[u, u] \approx \inf _{\substack{u \in \mathscr{U} \\\|u\|_{\mathscr{U}^{1,2}=1}}} D^{2} \Phi_{\mathrm{qc}}(y)[u, u]=: c_{\mathrm{qc}}(y) .
$$

If the approximate Hessian $D^{2} \Phi_{\mathrm{qc}}$ could be obtained by perturbing the coefficients of $D^{2} \Phi_{\mathrm{a}}$, then one could obtain such a result from an error estimate on the Hessians, that is, from a bound on $\left\|D^{2} \Phi_{\mathrm{a}}(y)-D^{2} \Phi_{\mathrm{qc}}(y)\right\|_{L\left(\mathscr{U}^{1,2}, \mathscr{U}^{-1,2}\right)}$. However, if we compute the two Hessians explicitly,

$$
\begin{gathered}
D^{2} \Phi_{\mathrm{a}}(y)[u, u]=\varepsilon \sum_{\xi=1}^{N} \phi^{\prime \prime}\left(y_{\xi}^{\prime}\right)\left|u_{\xi}^{\prime}\right|^{2}+\varepsilon \sum_{\xi=1}^{N} \phi^{\prime \prime}\left(y_{\xi}^{\prime}+y_{\xi+1}^{\prime}\right)\left|u_{\xi}^{\prime}+u_{\xi+1}^{\prime}\right|^{2}, \quad \text { and } \\
D^{2} \Phi_{\mathrm{qc}}(y)[u, u]=\varepsilon \sum_{\xi=1}^{N} \phi^{\prime \prime}\left(y_{\xi}^{\prime}\right)\left|u_{\xi}^{\prime}\right|^{2}+\varepsilon \sum_{\xi \in \mathcal{A}} \phi^{\prime \prime}\left(y_{\xi}^{\prime}+y_{\xi+1}^{\prime}\right)\left|u_{\xi}^{\prime}+u_{\xi+1}^{\prime}\right|^{2} \\
+\varepsilon \sum_{\xi \in \mathcal{C}} \frac{1}{2}\left\{\phi^{\prime \prime}\left(2 y_{\xi}^{\prime}\right)\left|2 u_{\xi}^{\prime}\right|^{2}+\phi^{\prime \prime}\left(2 y_{\xi+1}^{\prime}\right)\left|2 u_{\xi+1}^{\prime}\right|^{2}\right\},
\end{gathered}
$$

we observe that in the continuum region $D^{2} \Phi_{\mathrm{a}}(y)[u, u]$ contains nonzero coefficients for the mixed terms $u_{\xi}^{\prime} u_{\xi+1}^{\prime}$ which are replaced by diagonal terms in the QNLQC Hessian. This shows that it is impossible to obtain a useful estimate on the difference of the Hessians. Instead, we must aim to prove (4.1) directly.

The crucial observation, which is the basis of the stability analysis in this section, is that the nonlocal Hessian terms $\left|u_{\xi}^{\prime}+u_{\xi+1}^{\prime}\right|^{2}$ can be rewritten, by means of the polarisation identity, in terms of the local quantities $\left|u_{\xi}^{\prime}\right|^{2}$ and $\left|u_{\xi+1}^{\prime}\right|^{2}$ and a straingradient correction,

$$
\left|u_{\xi}^{\prime}+u_{\xi+1}^{\prime}\right|^{2}=2\left|u_{\xi}^{\prime}\right|^{2}+2\left|u_{\xi+1}^{\prime}\right|^{2}-\varepsilon^{2}\left|u_{\xi}^{\prime \prime}\right|^{2} .
$$


Using this simple formula it is straightforward to rewrite the Hessians in the form

$$
\begin{aligned}
D^{2} \Phi_{\mathrm{a}}(y)[u, u] & =\varepsilon \sum_{\xi=1}^{N} A_{\xi}\left|u_{\xi}^{\prime}\right|^{2}+\varepsilon \sum_{\xi=1}^{N} \varepsilon^{2} B_{\xi}\left|u_{\xi}^{\prime \prime}\right|^{2}, \text { and } \\
D^{2} \Phi_{\mathrm{qc}}(y)[u, u] & =\varepsilon \sum_{\xi=1}^{N} \tilde{A}_{\xi}\left|u_{\xi}^{\prime}\right|^{2}+\varepsilon \sum_{\xi \in \mathcal{A}} \varepsilon^{2} B_{\xi}\left|u_{\xi}^{\prime \prime}\right|^{2},
\end{aligned}
$$

where

$$
\begin{aligned}
& A_{\xi}=\phi^{\prime \prime}\left(y_{\xi}^{\prime}\right)+2 \phi^{\prime \prime}\left(y_{\xi-1}^{\prime}+y_{\xi}^{\prime}\right)+2 \phi^{\prime \prime}\left(y_{\xi}^{\prime}+y_{\xi+1}^{\prime}\right), \\
& \tilde{A}_{\xi}=\phi^{\prime \prime}\left(y_{\xi}^{\prime}\right)+\left\{\begin{aligned}
2 \phi^{\prime \prime}\left(y_{\xi-1}^{\prime}+y_{\xi}^{\prime}\right)+2 \phi^{\prime \prime}\left(y_{\xi}^{\prime}+y_{\xi+1}^{\prime}\right), & \xi \in \mathcal{A}^{\prime}, \\
2 \phi^{\prime \prime}\left(y_{\xi-1}^{\prime}+y_{\xi}^{\prime}\right)+2 \phi^{\prime \prime}\left(2 y_{\xi}^{\prime}\right), & \xi \in \mathcal{I}_{r}^{\prime}, \\
2 \phi^{\prime \prime}\left(y_{\xi}^{\prime}+y_{\xi+1}^{\prime}\right)+2 \phi^{\prime \prime}\left(2 y_{\xi}^{\prime}\right), & \xi \in \mathcal{I}_{\ell}^{\prime}, \\
4 \phi^{\prime \prime}\left(2 y_{\xi}^{\prime}\right), & \xi \in \mathcal{C}^{\prime} \backslash \mathcal{I}^{\prime},
\end{aligned}\right. \\
& B_{\xi}=-\phi^{\prime \prime}\left(y_{\xi}^{\prime}+y_{\xi+1}^{\prime}\right) .
\end{aligned}
$$

The proof of (4.3) requires purely algebraic manipulations.

We observe that, apart from estimating the effect of replacing $A_{\xi}$ by $\tilde{A}_{\xi}$, which is the purpose of the next lemma, our main challenge will be to understand the effect of dropping the strain gradient term in the continuum region.

Lemma 4.1. Let $y \in \mathscr{Y}$ such that $\min _{\xi \in \mathbb{Z}} y_{\xi}^{\prime}>0$, then

$$
\left\|A_{\xi}-\tilde{A}_{\xi}\right\|_{\ell \infty} \leq \varepsilon 4 \bar{C}_{3}\left\|y^{\prime \prime}\right\|_{\ell^{\infty}(\mathcal{C})},
$$

where $\bar{C}_{3}=C_{3}\left(2 \min _{\xi \in \mathcal{C}^{\prime}} y_{\xi}^{\prime}\right)$.

Proof. For $\xi \in \mathcal{A}^{\prime}, A_{\xi}=\tilde{A}_{\xi}$. For $\xi \in \mathcal{C}^{\prime} \backslash \mathcal{I}^{\prime}$, we have

$$
\begin{aligned}
A_{\xi}-\tilde{A}_{\xi} & =2\left(\phi^{\prime \prime}\left(y_{\xi-1}^{\prime}+y_{\xi}^{\prime}\right)-\phi^{\prime \prime}\left(2 y_{\xi}^{\prime}\right)\right)+2\left(\phi^{\prime \prime}\left(y_{\xi}^{\prime}+y_{\xi+1}^{\prime}\right)-\phi^{\prime \prime}\left(2 y_{\xi}^{\prime}\right)\right) \\
& =-\varepsilon 2 \phi^{\prime \prime \prime}\left(\vartheta_{1, \xi}\right) y_{\xi-1}^{\prime \prime}+\varepsilon 2 \phi^{\prime \prime \prime}\left(\vartheta_{2, \xi}\right) y_{\xi}^{\prime \prime},
\end{aligned}
$$

where $\vartheta_{1, \xi} \in \operatorname{conv}\left\{y_{\xi-1}^{\prime}+y_{\xi}^{\prime}, 2 y_{\xi}^{\prime}\right\}$ and $\vartheta_{2, \xi} \in \operatorname{conv}\left\{y_{\xi}^{\prime}+y_{\xi+1}^{\prime}, 2 y_{\xi}^{\prime}\right\}$. Hence, we obtain

$$
\left|A_{\xi}-\tilde{A}_{\xi}\right| \leq 2 \varepsilon \bar{C}_{3}\left|y_{\xi-1}^{\prime \prime}\right|+2 \varepsilon \bar{C}_{3}\left|y_{\xi}^{\prime \prime}\right| \leq 4 \varepsilon \bar{C}_{3}\left\|y^{\prime \prime}\right\|_{\ell \infty(\mathcal{C})} .
$$

Performing a similar calculation for the interfaces, and sacrificing a factor two there, we obtain the stated result.

Remark 4.2. If $\phi \in \mathrm{C}^{4}((0,+\infty])$, then the result of Lemma 4.1 can be improved. Using similar calculations as in the proof of Theorem 3.1, one obtains

$$
\left\|A_{\xi}-\tilde{A}_{\xi}\right\|_{\ell^{\infty}} \leq \max \left(\varepsilon 2 \bar{C}_{3}\left\|y^{\prime \prime}\right\|_{\ell^{\infty}(\mathcal{I})}, \varepsilon^{2} \bar{C}_{4}\left(\left\|y^{\prime \prime \prime}\right\|_{\ell^{\infty}\left(\mathcal{C}^{\prime}\right)}+\left\|y^{\prime \prime}\right\|_{\ell^{\infty}(\mathcal{C})}^{2}\right)\right) .
$$

Even though this result is clearly sharper than Lemma 4.1, it carries only limited practical value due to the fact that the maximum in $\left\|y^{\prime \prime}\right\|_{\ell_{\infty}(\mathcal{C})}$ will typically be attained at the interface.

Before formulating the first stability result, we briefly discuss the nature of the coefficients $B_{\xi}$. In view of the discussion in Section 2.3 we assume that $y_{\xi}^{\prime} \geq r_{*} / 2$ for all $\xi \in \mathbb{Z}$ and consequently $\phi^{\prime \prime}\left(y_{\xi}^{\prime}+y_{\xi+1}^{\prime}\right) \leq 0$ for all $\xi$, that is, we obtain the following result.

Lemma 4.3. If $y_{\xi}^{\prime} \geq r_{*} / 2$ for all $\xi \in \mathbb{Z}$, then

$$
B_{\xi} \geq 0 \quad \forall \xi \in \mathbb{Z} .
$$


4.2. An illustrative example. Before we turn to the stability estimates, we briefly discuss an example that further stresses the difference between atomistic and QC Hessians. We consider the case $y=F x$ and $\mathcal{A}=\emptyset$, that is, the QNL-QC method reduces to the local QC (or simply, Cauchy-Born) method. In that case, we have

$$
D^{2} \Phi_{\mathrm{a}}(F x)[u, u]=A\left\|u^{\prime}\right\|_{\ell_{\varepsilon}^{2}}^{2}+\varepsilon^{2} B\left\|u^{\prime \prime}\right\|_{\ell_{\varepsilon}^{2}} \quad \text { and } \quad D^{2} \Phi_{\mathrm{qc}}(F x)[u, u]=A\left\|u^{\prime}\right\|_{\ell_{\varepsilon}^{2}}^{2},
$$

where $A=\phi^{\prime \prime}(F)+4 \phi^{\prime \prime}(2 F)$ and $B=-\phi^{\prime \prime}(2 F)$.

In this case, the $\mathscr{U}^{1,2}$-spectrum of $D^{2} \Phi_{\mathrm{qc}}(F x)$ contains only the eigenvalue $A$ and every vector $u \in \mathscr{U}$ is an eigenvector. On the other hand, the $\mathscr{U}^{1,2}$-spectrum of $D^{2} \Phi_{\mathrm{a}}(F x)$ is given by

$$
\sigma_{\mathscr{U}^{1,2}}\left(D^{2} \Phi_{\mathrm{a}}(F x)\right)=\left\{A+B \mu_{j}: j=1, \ldots, N-1\right\}, \quad \text { where } \mu_{j}=4 \sin ^{2}\left(\frac{1}{2} j \pi \varepsilon\right) ;
$$

see [6, Sec. 3.1] and [7, Lemma 1] for similar analyses.

Thus, we see that the eigenvalues of the low eigenmodes are well approximated by the local QC stability constant $A$, and in particular,

$$
c_{\mathrm{a}}(F x)=A+4 B \sin ^{2}\left(\frac{1}{2} \pi \varepsilon\right)=A+O\left(\varepsilon^{2}\right)=c_{\mathrm{qc}}(F x)+O\left(\varepsilon^{2}\right) .
$$

On the other hand, the eigenvalues corresponding to the high frequency modes are significantly larger, in other words, high frequency perturbations are considerably more expensive in the atomistic model than in the continuum model. This discrepancy is not too surprising since the basic assumption of the Cauchy-Born model is the absence of high-frequency modes in the deformation.

Returning to the QNL-QC Hessian, we can observe from (4.3) that, if $F \geq r_{*} / 2$, then $c_{\mathrm{qc}}(F x)=A$ as well, and hence (4.5) holds independently of the choice of the atomistic region. This observation was one of the main results in [6].

4.3. An a priori stability result. A common approach to establish the stability of a QC method is to assume that next-nearest neighbour interactions are dominated by nearest-neighbour interactions $4,14,18,21$. This leads to particularly simple results that can even be made fairly sharp as the numerical experiments in 21 demonstrate. If one wishes to include more than the simplest examples in such stability results, then one should specify classes of atomistic solutions that are of interest and prove, for each such class, that the QC Hessian is stable when evaluated at those deformations. In [21, two main types of stable solutions were identified for a variant of the model problem (2.3): (i) elastic deformations and (ii) deformations with a single crack. Here we will focus only on elastic solutions.

Proposition 4.4 (Stability of elastic states). Let $y \in \mathscr{Y}$ such that $y_{\xi}^{\prime} \geq r_{*} / 2$ for all $\xi \in \mathbb{Z}$, then

$$
c_{a}(y) \geq \min _{\xi=1, \ldots, N} A_{\xi}, \quad \text { and } \quad c_{q c}(y) \geq \min _{\xi=1, \ldots, N} \tilde{A}_{\xi},
$$

where $c_{a}(y), c_{q c}(y)$ are defined in (4.1) and $A_{\xi}, \tilde{A}_{\xi}$ are defined in (4.3).

Proof. Both bounds in (4.6) follow immediately from (4.3) and from the fact that $B_{\xi} \geq 0$ for all $\xi \in \mathbb{Z}$.

Remark 4.5. Proposition 4.4 provides a particularly simple example of atomistic configurations for which the QNL-QC method is stable. Due to the representation of next-nearest neighbour interactions developed in Section 4.1 these results are sharper than those found in [4, 18, 21, and will be sufficient whenever elastic 
effects dominate. Since there is no regularity assumption on $y$, simple singularities are included as well.

Remark 4.6. It must be stressed at this point that the stated goal, namely a proof of $c_{\mathrm{a}}(y) \approx c_{\mathrm{qc}}(y)$, or at least $c_{\mathrm{qc}}(y) \gtrsim c_{\mathrm{a}}(y)$, up to a controllable error, has not been obtained here. We observe, however, that our lower bound on the QNL-QC coercivity constant is exact when evaluated at a homogeneous deformation. More precisely, let $\underline{c}_{\mathrm{qc}}(y)=\min _{\xi=1, \ldots, N} \tilde{A}_{\xi}$, then $\underline{c}_{\mathrm{qc}}(F x)=c_{\mathrm{qc}}(F x)$ for all $F \geq r_{*} / 2$. It is therefore reasonable to expect that the result of Proposition 4.4 is fairly sharp.

A result of the kind $c_{\mathrm{qc}}(y) \gtrsim c_{\mathrm{a}}(y)$ for more general deformations $y$ can be related to the regularity of eigenfunctions in the continuum region. Namely, let $\bar{u}$ be an eigenfunction for $D^{2} \Phi_{\mathrm{qc}}(y)$ associated with the eigenvalue $c_{\mathrm{qc}}(y)$, then

$$
\begin{aligned}
c_{\mathrm{a}}(y) & =\min _{\left\|u^{\prime}\right\|_{\ell_{\varepsilon}^{2}=1}} D^{2} \Phi_{\mathrm{a}}(y)[u, u] \\
& \leq D^{2} \Phi_{\mathrm{a}}(y)[\bar{u}, \bar{u}] \\
& \leq c_{\mathrm{qc}}(y)+\left|D^{2} \Phi_{\mathrm{a}}(y)[\bar{u}, \bar{u}]-D^{2} \Phi_{\mathrm{qc}}(y)[\bar{u}, \bar{u}]\right| .
\end{aligned}
$$

Using (4.3) and Lemma 4.1, we obtain the estimate

$$
\left|D^{2} \Phi_{\mathrm{a}}(y)[\bar{u}, \bar{u}]-D^{2} \Phi_{\mathrm{qc}}(y)[\bar{u}, \bar{u}]\right| \leq 4 \bar{C}_{3} \varepsilon\left\|y^{\prime \prime}\right\|_{\ell^{\infty}(\mathcal{C})}+\varepsilon^{3} \sum_{\xi \in \mathcal{C}} B_{\xi}\left|\bar{u}_{\xi}^{\prime \prime}\right|^{2} .
$$

Thus, if one could establish an $O\left(\varepsilon^{-1 / 2}\right)$ bound on $\left\|\bar{u}^{\prime \prime}\right\|_{\ell_{\varepsilon}^{2}(\mathcal{C})}$, for example, in terms of the smoothness of $y$ in the continuum region, then one would obtain

$$
c_{\mathrm{qc}}(y) \geq c_{\mathrm{a}}(y)-O(\varepsilon) .
$$

However, obtaining such a bound is technical even in one dimension.

4.4. A posteriori stability. If we assume that $y_{\mathrm{qc}}$ is a local minimizer of the QNL-QC approximation, then we can check the condition whether $D^{2} \Phi_{\mathrm{qc}}\left(y_{\mathrm{qc}}\right)>0$ a posteriori by solving a (generalized) eigenvalue problem. The question we then ought to ask is whether this stability carries over to the full atomistic model, that is, whether also $D^{2} \Phi_{\mathrm{a}}\left(y_{\mathrm{qc}}\right)>0$. If this were not the case, then it cannot be guaranteed that a local minimizer in the QNL-QC model corresponds to a local minimizer in the atomistic model. However, for our model problem the answer is surprisingly simple and is given in the following result.

Theorem 4.7 (A posteriori stability). Let $y \in \mathscr{Y}$ such that $\min _{\xi \in \mathbb{Z}} y_{\xi}^{\prime}>0$, and such that

$$
\phi^{\prime \prime}\left(y_{\xi}^{\prime}+y_{\xi+1}^{\prime}\right) \leq 0 \quad \forall \xi \in \mathcal{C},
$$

then

$$
c_{a}(y) \geq c_{q c}(y)-\varepsilon 4 \bar{C}_{3}\left\|y^{\prime \prime}\right\|_{\ell^{\infty}(\mathcal{C})},
$$

where $\bar{C}_{3}=C_{3}\left(2 \min _{\xi \in \mathcal{C}^{\prime}} y_{\xi}^{\prime}\right)$.

Proof. From Lemma 4.1 and the assumption that $\phi^{\prime \prime}\left(y_{\xi}^{\prime}+y_{\xi+1}^{\prime}\right) \leq 0$, or equivalently, $B_{\xi} \geq 0$ for all $\xi \in \mathcal{C}$ we have, for all $u \in \mathscr{U}$,

$$
D^{2} \Phi_{\mathrm{a}}(y)[u, u] \geq D^{2} \Phi_{\mathrm{qc}}(y)[u, u]-\varepsilon 4 \bar{C}_{3}\left\|y^{\prime \prime}\right\|_{\ell^{\infty}(\mathcal{C})}\left\|u^{\prime}\right\|_{\ell_{\varepsilon}^{2}}^{2} .
$$

The stated result follows by taking the infimum over $u \in \mathscr{U}$ with $\|u\|_{\mathscr{U}^{1,2}}=1$. 
Remark 4.8. Theorem 4.7 is false in higher dimensions. It is possible that a QC model (for example, a pure Cauchy-Born model) is stable at a uniform deformation $F x$ while the atomistic model is unstable [11, Sec. 4.2]. Thus, at the very least, one needs to add an additional assumption that the deformation gradients in the continuum region belong to an atomistic stability regime.

4.5. A remark on defects. Suppose now that a deformation $y \in \mathscr{Y}$ has a single "crack", that is, there exists a single index $\hat{\xi}$ such that $y_{\hat{\xi}}^{\prime}=O(N)$. Assume, for example, that $F>1$ and that $\hat{y} \in \mathscr{Y}$ is given by

$$
\hat{y}_{\xi}^{\prime}=\left\{\begin{aligned}
1, & \text { if } \xi \neq \hat{\xi} \\
F+(N-1)(F-1), & \text { otherwise. }
\end{aligned}\right.
$$

If $N$ is sufficiently large, then $A_{\hat{\xi}} \leq 0$ and hence (4.6) gives no useful information. This is no accident as the following result demonstrates.

Proposition 4.9. Suppose that $r_{*}>1$ and that there exists a cut-off radius $r_{c}>r_{*}$ such that $\phi=0$ in $\left[r_{c},+\infty\right)$. Let $\hat{y} \in \mathscr{Y}$ be defined by (4.7), then, for sufficiently large $N$,

$$
c_{a}(\hat{y}) \leq 2 \hat{A} \varepsilon \quad \text { and } \quad c_{q c}(\hat{y}) \leq 2 \hat{A} \varepsilon, \quad \text { where } \quad \hat{A}=\phi^{\prime \prime}(1)+4 \phi^{\prime \prime}(2) .
$$

Proof. There exists a unique test function $\hat{u} \in \mathscr{U}$ such that

$$
\hat{u}_{\xi}^{\prime}=\left\{\begin{aligned}
(N-1)^{1 / 2}, & \xi=\hat{\xi} \\
-(N-1)^{-1 / 2}, & \xi \neq \hat{\xi}
\end{aligned}\right.
$$

and we note that $\left\|\hat{u}_{\xi}^{\prime}\right\|_{\ell_{\varepsilon}^{2}}=1$.

Next, we observe that $\hat{u}_{\xi}^{\prime \prime}=0$ for all $\xi$ except for $\xi=\hat{\xi}, \hat{\xi}-1$. However, since $\phi=0$ in $\left[r_{c},+\infty\right)$, it follows that $B_{\hat{\xi}-1}=B_{\hat{\xi}}=0$ for $N$ sufficiently large, and hence we can simply ignore the strain gradient term. Thus, testing $D^{2} \Phi_{\mathrm{a}}(\hat{y})$ with $\hat{u}$, we obtain

$$
D^{2} \Phi_{\mathrm{a}}(\hat{y})[\hat{u}, \hat{u}]=\varepsilon \sum_{\xi=1}^{N} A_{\xi}(N-1)^{-1} .
$$

Setting $\hat{A}=\phi^{\prime \prime}(1)+4 \phi^{\prime \prime}(2)$, we have $A_{\xi}=\hat{A}$ for all $\xi$ except $\xi=\hat{\xi}-1, \hat{\xi}, \hat{\xi}+1$. For $\xi=\hat{\xi}$, we have $A_{\hat{\xi}} \leq 0$ provided that $N$ is sufficiently large. Combining $A_{\hat{\xi} \pm 1}$ we estimate

$$
A_{\hat{\xi}+1}+A_{\hat{\xi}-1}=2 \phi^{\prime \prime}(1)+4 \phi^{\prime \prime}(2)=\hat{A}+\phi^{\prime \prime}(1) .
$$

Hence, we obtain that, if $N$ is sufficiently large,

$$
D^{2} \Phi_{\mathrm{a}}(\hat{y})[\hat{u}, \hat{u}]=\varepsilon \sum_{\xi=1}^{N} A_{\xi}(N-1)^{-1} \leq \varepsilon \hat{A}+\varepsilon(N-1)^{-1} \phi^{\prime \prime}(1) \leq 2 \varepsilon \hat{A} .
$$

Since $c_{\mathrm{a}}(\hat{y}) \geq c_{\mathrm{qc}}(\hat{y})$ for this example, the same result also holds for the QC stability constant.

Since deformations with cracks or other defects can surely be stable equilibria, this result only shows that they cannot be analyzed in the $\mathscr{U}^{1,2}$ space. Different topologies must be chosen to analyze the stability of defects. The analysis in 21] suggests that the correct norm for a 1D example with a single crack is

$$
\|u\|_{\mathscr{U}_{\hat{\xi}}^{1,2}}:=\left(\varepsilon \sum_{\xi \neq \hat{\xi}}\left|u_{\xi}^{\prime}\right|^{2}\right)^{1 / 2}
$$


however, we will not pursue this direction further in the present work. We note, however, that changing the norm inside the atomistic region does not change the residual estimate. To see this, one verifies that, in the last line of the proof of Theorem 3.1, the term $\left\|u^{\prime}\right\|_{\ell_{\varepsilon}^{p^{\prime}}}$ may be replaced by $\left\|u^{\prime}\right\|_{\ell_{\varepsilon}^{p^{\prime}}\left(\mathcal{C}^{\prime}\right)}$. This suggests that one could use coercivity estimates of the type

$$
D^{2} \Phi_{\mathrm{qc}}(y)[u, u] \geq \tilde{c}_{\mathrm{qc}}(y)\left\|u^{\prime}\right\|_{\ell_{\varepsilon}^{2}\left(\mathcal{C}^{\prime}\right)}^{2}
$$

in the error analysis. However, this would not allow us to pursue the rigorous nonlinear analysis in the next section.

\section{EXISTENCE AND ERROR ESTIMATES}

In this section, we will combine the consistency and stability analysis of Sections 3 and 4 to prove a priori and a posteriori existence results and error estimates.

5.1. A priori analysis. In this section, we extend the main results in [5, 18, to the context of large deformations and solutions that are possibly nonsmooth in the atomistic region.

We begin by assuming the existence of a strongly stable equilibrium $y_{\mathrm{a}}$ of the full atomistic model. If we assume that this deformation is "smooth" in the continuum region, then Theorem 3.1 shows that the truncation error is small, and hence $y_{\mathrm{a}}$ can be considered an approximate solution of the QNL-QC equilibrium equations (2.8). If $y_{\mathrm{a}}$ is also stable in the QNL-QC model (i.e., $D^{2} \Phi_{\mathrm{qc}}\left(y_{\mathrm{a}}\right)$ is positive definite), then we can employ the inverse function theorem, Lemma 2.2, to prove the existence of a solution $y_{\mathrm{qc}}$ of the QNL-QC equilibrium equations in a small neighbourhood of $y_{\mathrm{a}}$. This idea is made rigorous in the following result, which is best read as follows:

"If $y_{a}$ is a strongly stable local minimizer of $\Phi_{a}^{\text {tot }}$, which is smooth in the continuum region, then there exists a solution $y_{q c}$ of the QNL-QC method, which is a good approximation to $y_{a}$."

Theorem 5.1. Let $y_{a} \in \operatorname{argmin} \Phi_{a}^{t o t}$, and assume that $y_{a, \xi}^{\prime} \geq r_{*} / 2$, and that

$$
\min _{\xi=1, \ldots, N} A_{\xi}=: \underline{A}>0 .
$$

Then there exist constants $\delta_{1}, \delta_{2}$ depending only on $\min _{\xi} y_{a, \xi}^{\prime}$ and on $\underline{A}$, such that, if

$$
\begin{aligned}
\varepsilon\left\|y_{a}^{\prime \prime}\right\|_{\ell^{\infty}(\mathcal{C})} & \leq \delta_{1}, \quad \text { and } \\
\varepsilon^{1 / 2}\left\|y_{a}^{\prime \prime}\right\|_{\ell_{\varepsilon}^{2}(\mathcal{I})}+\varepsilon^{3 / 2}\left(\left\|y_{a}^{\prime \prime \prime}\right\|_{\ell_{\varepsilon}^{2}\left(\mathcal{C}^{\prime} \backslash \mathcal{I}^{\prime}\right)}+\left\|y_{a}^{\prime \prime}\right\|_{\ell_{\varepsilon}^{4}(\mathcal{C})}^{2}\right) & \leq \delta_{2},
\end{aligned}
$$

then there exists a (locally unique) local minimizer $y_{q c}$ of $\Phi_{q c}^{\text {tot }}$ in $\mathscr{Y}$ such that

$$
\left\|\left(y_{a}-y_{q c}\right)^{\prime}\right\|_{\ell_{\varepsilon}^{2}} \leq 4 \underline{A}^{-1}\left\{\varepsilon \bar{C}_{2}\left\|y_{a}^{\prime \prime}\right\|_{\ell_{\varepsilon}^{2}(\mathcal{I})}+\varepsilon^{2} \bar{C}_{3}\left(\left\|y_{a}^{\prime \prime \prime}\right\|_{\ell_{\varepsilon}^{2}\left(\mathcal{C}^{\prime} \backslash \mathcal{I}^{\prime}\right)}+\left\|y_{a}^{\prime \prime}\right\|_{\ell_{\varepsilon}^{4}(\mathcal{C})}^{2}\right)\right\} .
$$

where $\bar{C}_{i}=C_{i}\left(2 \min _{\xi \in \mathcal{C}^{\prime}} y_{a, \xi}^{\prime}\right), i=2,3$.

Proof. To put the statement into the context of Lemma 2.2 we define $X=\mathscr{U}^{1,2}$, $Y=\mathscr{U}^{-1,2}, \mathscr{F}(w)=D \Phi_{\mathrm{qc}}^{\text {tot }}\left(y_{\mathrm{a}}+w\right)$, and $x_{0}=0$. The set $A$ is given by

$$
A=\left\{w \in \mathscr{U}: y_{a, \xi}^{\prime}+w_{\xi}^{\prime}>0, \xi \in \mathbb{Z}\right\}
$$

Since $\phi \in \mathrm{C}^{3}((0,+\infty])$, it is clear that $\mathscr{F}$ is continuously (Fréchet) differentiable, with $D \mathscr{F}(0)=D^{2} \Phi_{\mathrm{qc}}\left(y_{\mathrm{a}}\right)$. 
1. Consistency: From Theorem 3.1 we obtain that

$$
\begin{aligned}
\|\mathscr{F}(0)\|_{Y} & =\left\|D \Phi_{\mathrm{qc}}^{\mathrm{tot}}\left(y_{\mathrm{a}}\right)\right\|_{\mathscr{U}^{-1,2}}=\left\|D \Phi_{\mathrm{qc}}\left(y_{\mathrm{a}}\right)-D \Phi_{\mathrm{a}}\left(y_{\mathrm{a}}\right)\right\|_{\mathscr{U}^{-1,2}} \\
& \leq \varepsilon \bar{C}_{2}\left\|y_{\mathrm{a}}^{\prime \prime \prime}\right\|_{\ell_{\varepsilon}^{2}(\mathcal{I})}+\varepsilon^{2} \bar{C}_{3}\left(\left\|y_{\mathrm{a}}^{\prime \prime \prime}\right\|_{\ell_{\varepsilon}^{2}\left(\mathcal{C}^{\prime} \backslash \mathcal{I}^{\prime}\right)}+\left\|y_{\mathrm{a}}^{\prime \prime}\right\|_{\ell_{\varepsilon}^{4}(\mathcal{C})}^{2}\right)=: \eta .
\end{aligned}
$$

2. Stability: The assumption that $y_{a, \xi}^{\prime} \geq r_{*} / 2$ implies that $B_{\xi} \geq 0$ for all $\xi$. Using this fact, the "elasticity assumption" (5.1), and Lemma 4.1, we obtain

$$
\begin{aligned}
D^{2} \Phi_{\mathrm{qc}}\left(y_{\mathrm{a}}\right)[u, u] & \geq \varepsilon \sum_{\xi=1}^{N} \tilde{A}_{\xi}\left|u_{\xi}^{\prime}\right|^{2} \\
& \geq \varepsilon \sum_{\xi=1}^{N} A_{\xi}\left|u_{\xi}^{\prime}\right|^{2}-\varepsilon 4 \bar{C}_{3}\left\|y_{\mathrm{a}}^{\prime \prime}\right\|_{\ell^{\infty}(\mathcal{C})}\left\|u^{\prime}\right\|_{\ell_{\varepsilon}^{2}(\mathcal{C})}^{2} \\
& \geq\left(\underline{A}-\varepsilon 4 \bar{C}_{3}\left\|y_{\mathrm{a}}^{\prime \prime}\right\|_{\ell^{\infty}(\mathcal{C})}\right)\left\|u^{\prime}\right\|_{\ell_{\varepsilon}^{2}}^{2} .
\end{aligned}
$$

Hence, we obtain

$$
c_{\mathrm{qc}}\left(y_{\mathrm{a}}\right) \geq \underline{A}-\varepsilon 4 \bar{C}_{3}\left\|y_{\mathrm{a}}^{\prime \prime}\right\|_{\ell \infty(\mathcal{C})},
$$

and consequently, if $\underline{A}-\varepsilon 4 \bar{C}_{3}\left\|y_{\mathrm{a}}^{\prime \prime}\right\|_{\ell \infty(\mathcal{C})}>0$, then

$$
\left\|D \mathscr{F}(0)^{-1}\right\|_{L\left(\mathscr{U}-1,2, \mathscr{U}^{1,2}\right)} \leq\left(\underline{A}-\varepsilon 4 \bar{C}_{3}\left\|y_{\mathrm{a}}^{\prime \prime}\right\|_{\ell^{\infty}(\mathcal{C})}\right)^{-1} \text {. }
$$

Setting $\delta_{1}=\underline{A} /\left(8 \bar{C}_{3}\right)$, (5.2) becomes

$$
\varepsilon 4 \bar{C}_{3}\left\|y_{\mathrm{a}}^{\prime \prime}\right\|_{\ell^{\infty}(\mathcal{C})} \leq \frac{1}{2} \underline{A}
$$

and hence we obtain that

$$
\left\|D \mathscr{F}(0)^{-1}\right\|_{L\left(\mathscr{U}^{-1,2}, \mathscr{U}^{1,2}\right)} \leq \sigma:=2 / \underline{A} .
$$

3. Lipschitz bound: Next, we compute a Lipschitz bound for $D \mathscr{F}=D^{2} \Phi_{\mathrm{qc}}$. To this end, we first need to establish a lower bound on $y_{\mathrm{a}, \xi}^{\prime}+w_{\xi}^{\prime}$ for all $w$ satisfying $\left\|w^{\prime}\right\|_{\ell_{\varepsilon}^{2}} \leq 2 \eta \sigma$. Since the $\mathscr{U}^{1,2}$-topology cannot provide this bound directly, we need to resort to an inverse inequality: for all $w \in \mathscr{U}$ such that $\|w\|_{\mathscr{U}^{1,2}} \leq 2 \eta \sigma$, we have

$$
\left\|w^{\prime}\right\|_{\ell^{\infty}} \leq \varepsilon^{-1 / 2}\left\|w^{\prime}\right\|_{\ell_{\varepsilon}^{2}} \leq M_{1}^{\prime}\left(\varepsilon^{1 / 2}\left\|y_{\mathrm{a}}^{\prime \prime}\right\|_{\ell_{\varepsilon}^{2}(\mathcal{I})}+\varepsilon^{3 / 2}\left(\left\|y_{\mathrm{a}}^{\prime \prime \prime}\right\|_{\ell_{\varepsilon}^{2}\left(\mathcal{C}^{\prime} \backslash \mathcal{I}^{\prime}\right)}+\left\|y_{\mathrm{a}}^{\prime \prime}\right\|_{\ell_{\varepsilon}^{4}(\mathcal{C})}^{2}\right)\right),
$$

where $M_{1}^{\prime}$ is a constant that depends only on $\underline{A}$ and on $\bar{C}_{2}, \bar{C}_{3}$. Hence, if

$$
\varepsilon^{1 / 2}\left\|y_{\mathrm{a}}^{\prime \prime}\right\|_{\ell_{\varepsilon}^{2}(\mathcal{I})}+\varepsilon^{3 / 2}\left(\left\|y_{\mathrm{a}}^{\prime \prime \prime}\right\|_{\ell_{\varepsilon}^{2}\left(\mathcal{C}^{\prime} \backslash \mathcal{I}^{\prime}\right)}+\left\|y_{\mathrm{a}}^{\prime \prime}\right\|_{\ell_{\varepsilon}^{4}(\mathcal{C})}^{2}\right) \leq \frac{1-\delta}{M_{1}^{\prime}} \min _{\xi} y_{\mathrm{a}, \xi}^{\prime},
$$

for some $\delta \in(0,1)$, then the desired bound

holds.

$$
y_{\mathrm{a}, \xi}^{\prime}+w_{\xi}^{\prime} \geq \delta y_{\mathrm{a}, \xi}^{\prime}
$$

It is now a simple exercise to show that, for any two displacements $w_{1}, w_{2} \in \mathscr{U}$, such that $\left\|w_{i}\right\|_{\mathscr{U}^{1,2}} \leq 2 \eta \sigma$, we have the Lipschitz bound

$$
\begin{array}{r}
\left|D^{2} \Phi_{\mathrm{qc}}\left(y_{\mathrm{a}}+w_{1}\right)[u, v]-D^{2} \Phi_{\mathrm{qc}}\left(y_{\mathrm{a}}+w_{2}\right)[u, v]\right| \leq L^{\prime}\left\|\left(w_{1}-w_{2}\right)^{\prime}\right\|_{\ell^{\infty}}\left\|u^{\prime}\right\|_{\ell_{\varepsilon}^{2}}\left\|v^{\prime}\right\|_{\ell_{\varepsilon}^{2}} \\
\forall u, v \in \mathscr{U},
\end{array}
$$

where $L^{\prime}$ is a constant that depends only $C_{3}\left(\delta \min _{\xi} y_{\mathrm{a}, \xi}^{\prime}\right)$. Applying the same inverse inequality as in (5.5) we obtain that

$$
\left\|D \mathscr{F}\left(w_{1}\right)-D \mathscr{F}\left(w_{2}\right)\right\|_{L\left(\mathscr{U}^{1,2}, \mathscr{U}^{-1,2}\right)} \leq \varepsilon^{-1 / 2} L^{\prime}\left\|w_{1}-w_{2}\right\|_{\mathscr{U}^{1,2}} .
$$


We set $L=\varepsilon^{-1 / 2} L^{\prime}$. (At this point we could choose $\delta$ in order to optimize the size of $L$ against the requirement (5.6). Since we are only interested in a qualitative result, we will ignore this possibility.)

4. Conclusion: To conclude the proof, we only need to check the condition under which $2 L \sigma^{2} \eta<1$. Inserting the bounds for the various terms, assuming that (55.6) and (5.4) hold, we obtain that

$$
2 L \sigma^{2} \eta \leq M_{2}^{\prime}\left\{\varepsilon^{1 / 2}\left\|y_{\mathrm{a}}^{\prime \prime}\right\|_{\ell_{\varepsilon}^{2}(\mathcal{I})}+\varepsilon^{3 / 2}\left(\left\|y_{\mathrm{a}}^{\prime \prime \prime}\right\|_{\ell_{\varepsilon}^{2}\left(\mathcal{C}^{\prime} \backslash \mathcal{I}^{\prime}\right)}+\left\|y_{\mathrm{a}}^{\prime \prime}\right\|_{\ell_{\varepsilon}^{4}(\mathcal{C})}^{2}\right)\right\}
$$

where $M_{2}^{\prime}$ depends only on $\underline{A}$, on $\bar{C}_{2}, \bar{C}_{3}$, and on $L^{\prime}$.

Hence, we conclude that, if (5.2) and (5.3) hold with

$$
\delta_{1}=\underline{A} /\left(8 \bar{C}_{3}\right) \quad \text { and } \quad \delta_{2}=\min \left\{\frac{1-\delta}{M_{1}^{\prime}} \min _{\xi} y_{\mathrm{a}, \xi}^{\prime}, 1 / M_{2}^{\prime}\right\},
$$

then there exists $w_{\mathrm{qc}} \in \mathscr{U}$ such that $F\left(w_{\mathrm{qc}}\right)=0$, or equivalently $D \Phi_{\mathrm{qc}}^{\mathrm{tot}}\left(y_{\mathrm{a}}+w_{\mathrm{qc}}\right)=$ 0 , and

$$
\left\|w_{\mathrm{qc}}\right\|_{\mathscr{U}^{1,2}} \leq 2 \eta \sigma \leq \underline{\underline{A}} \eta .
$$

Taking into account the bound for $\eta$ from step 1, we obtain the stated error estimate.

Local minimality of $y_{\mathrm{qc}}=y_{\mathrm{a}}+w_{\mathrm{qc}}$ follows from (5.2) which implies that

$$
c_{\mathrm{qc}}\left(y_{\mathrm{a}}\right) \geq \frac{1}{2} \underline{A},
$$

and the local Lipschitz bound, which guarantees

$$
c_{\mathrm{qc}}\left(y_{\mathrm{qc}}\right) \geq c_{\mathrm{qc}}\left(y_{\mathrm{a}}\right)-L(2 \eta \sigma)>c_{\mathrm{qc}}\left(y_{\mathrm{a}}\right)-\frac{1}{\sigma}=0 .
$$

Thus $D^{2} \Phi_{\mathrm{qc}}\left(y_{\mathrm{qc}}\right)$ is positive definite and therefore $y_{\mathrm{qc}}$ is a local minimizer.

Remark 5.2. As indicated above, Theorem 5.1 is a variation or generalization of [5. Thm. 4.1] and [18, Thm. 5.13], and hence we should briefly discuss the differences between these results.

Theorem 4.1 in [5] treats a linearized case, but is otherwise fairly sharp. For example, the stability condition derived in [5, Lemma 2.3] was later shown to be exact [6]. The convergence rate in terms of the atomic spacing $\varepsilon$ (or $h$ in [5]) is optimal, however, the dependence of the error on the smoothness of the exact solution is much stronger than in Theorem 5.1. This is a consequence of deriving the trunction error estimate in an $\ell^{p}$-type norm, and comparing the $\mathrm{QC}$ solution with a continuum solution instead of the exact atomistic solution.

Although a very different terminology is used, some aspects of the proof of [18, Thm. 5.13] are quite similar to the analysis presented here. However, in [18 the consistency analysis is based on a higher order expansion of the atomistic solution in terms of the Cauchy-Born model. As a result, the analysis proves the existence of atomistic and QC solutions only for "sufficiently small and sufficiently smooth" applied forces, in a small neighbourhood of the reference state $F x$. Moreover, the use of a different norm for measuring the truncation error [18, Eq. (5.27)] leads to error estimates that are optimal in $\varepsilon$ only in the $\mathscr{U}^{1, \infty}$-norm, and it is unclear from the presentation how the error depends on the smoothness of the atomistic solution.

The work in [18] is more general than the present paper in that it analyzes the more general geometrically consistent $Q C$ scheme proposed in [10]. 
5.2. A posteriori analysis. Theorem 5.1 is an a priori result, that is, it guarantees the existence and accuracy of a QNL-QC solution under certain assumptions on a given atomistic solution. Results of this type can guarantee that certain classes of atomistic solutions can be reliably approximated by the QNL-QC method. In practise, however, an a posteriori result of the same type may be even more interesting. Given a computed QNL-QC solution $y_{\mathrm{qc}}$, we may ask whether an (exact) atomistic solution $y_{\mathrm{a}}$ exists to which $y_{\mathrm{qc}}$ is an approximation. Questions of this kind are investigated in the literature on numerical enclosure methods (see [23] for a recent review, or [20] where this concept is called a posteriori existence) and leads to the following result, which is best read as follows:

"If $y_{q c}$ is a strongly stable QNL-QC solution, which is smooth in the continuum region, then there exists an exact solution $y_{a}$ of the full atomistic model, for which $y_{q c}$ is a good approximation."

Theorem 5.3. Let $y_{q c} \in \operatorname{argmin} \Phi_{q c}^{\text {tot }}$, such that $c_{q c}\left(y_{q c}\right)>0$ and

$$
\phi^{\prime \prime}\left(\left(y_{q c}\right)_{\xi}^{\prime}+\left(y_{q c}\right)_{\xi+1}^{\prime}\right) \leq 0 \quad \forall \xi \in \mathcal{C} .
$$

Then there exist constants $\delta_{1}, \delta_{2}$ that depend only on $\min _{\xi}\left(y_{q c}\right)_{\xi}^{\prime}$ and on $c_{q c}\left(y_{q c}\right)$ such that, if

$$
\begin{aligned}
\varepsilon\left\|y_{q c}^{\prime \prime}\right\|_{\ell^{\infty}(\mathcal{C})} & \leq \delta_{1}, \quad \text { and } \\
\varepsilon^{1 / 2}\left\|y_{q c}^{\prime \prime}\right\|_{\ell_{\varepsilon}^{2}(\mathcal{I})}+\varepsilon^{3 / 2}\left(\left\|y_{q c}^{\prime \prime \prime}\right\|_{\ell_{\varepsilon}^{2}\left(\mathcal{C}^{\prime} \backslash \mathcal{I}^{\prime}\right)}+\left\|y_{q c}^{\prime \prime}\right\|_{\ell_{\varepsilon}^{4}(\mathcal{C})}^{2}\right) & \leq \delta_{2},
\end{aligned}
$$

then there exists a strongly stable local minimizer $y_{a}$ of $\Phi_{a}^{\text {tot }}$ in $\mathscr{Y}$ such that

$$
\left\|\left(y_{q c}-y_{a}\right)^{\prime}\right\|_{\ell_{\varepsilon}^{2}} \leq 2 \frac{\varepsilon \bar{C}_{2}\left\|y_{q c}^{\prime \prime}\right\|_{\ell_{\varepsilon}^{2}(\mathcal{I})}+\varepsilon^{2} \bar{C}_{3}\left(\left\|y_{q c}^{\prime \prime \prime}\right\|_{\ell_{\varepsilon}^{2}\left(\mathcal{C}^{\prime} \backslash \mathcal{I}^{\prime}\right)}+\left\|y_{q c}^{\prime \prime}\right\|_{\ell_{\varepsilon}^{4}(\mathcal{C})}^{2}\right)}{c_{q c}\left(y_{q c}\right)-\varepsilon 4 \bar{C}_{3}\left\|y_{q c}^{\prime \prime}\right\|_{\ell^{\infty}(\mathcal{C})}},
$$

where $\bar{C}_{i}=C_{i}\left(2 \min _{\xi \in \mathcal{C}^{\prime}}\left(y_{q c}\right)_{\xi}^{\prime}\right), i=2,3$.

Proof. The proof follows along very similar lines as the proof of Theorem 5.1, and we will therefore only focus on those parts of the argument that change.

To put the statement into the context of Lemma 2.2, we define $X=\mathscr{U}^{1,2}, Y=$ $\mathscr{U}^{-1,2}, \mathscr{F}(w)=D \Phi_{\mathrm{a}}^{\mathrm{tot}}\left(y_{\mathrm{qc}}+w\right)$, and $x_{0}=0$. The set $A$ is again given by

$$
A=\left\{w \in \mathscr{U}:\left(y_{\mathrm{qc}}\right)_{\xi}^{\prime}+w_{\xi}^{\prime}>0, \xi \in \mathbb{Z}\right\} .
$$

Since $\phi \in \mathrm{C}^{3}(0,+\infty)$, it follows that $\mathscr{F}$ is continuously Fréchet differentiable, with $D \mathscr{F}(0)=D^{2} \Phi_{\mathrm{a}}\left(y_{\mathrm{qc}}\right)$.

1. Consistency: From Theorem 3.1, we obtain

$$
\begin{aligned}
\|\mathscr{F}(0)\|_{Y} & =\left\|D \Phi_{\mathrm{a}}^{\mathrm{tot}}\left(y_{\mathrm{qc}}\right)\right\|_{\mathscr{U}^{-1,2}}=\left\|D \Phi_{\mathrm{a}}\left(y_{\mathrm{qc}}\right)-D \Phi_{\mathrm{qc}}\left(y_{\mathrm{qc}}\right)\right\|_{\mathscr{U}^{-1,2}} \\
& \leq \varepsilon \bar{C}_{2}\left\|y_{\mathrm{qc}}^{\prime \prime}\right\|_{\ell_{\varepsilon}^{2}(\mathcal{I})}+\varepsilon^{2} \bar{C}_{3}\left(\left\|y_{\mathrm{qc}}^{\prime \prime \prime}\right\|_{\ell_{\varepsilon}^{2}\left(\mathcal{C}^{\prime} \backslash \mathcal{I}^{\prime}\right)}+\left\|y_{\mathrm{qc}}^{\prime \prime}\right\|_{\ell_{\varepsilon}^{4}(\mathcal{C})}^{2}\right)=: \eta .
\end{aligned}
$$

2. Stability: Using Theorem 4.7, we obtain

$$
c_{\mathrm{a}}\left(y_{\mathrm{qc}}\right) \geq c_{\mathrm{qc}}\left(y_{\mathrm{qc}}\right)-\varepsilon 4 \bar{C}_{3}\left\|y_{\mathrm{qc}}^{\prime \prime}\right\|_{\ell^{\infty}(\mathcal{C})}=: 1 / \sigma,
$$

where $\bar{C}_{3}=C_{3}\left(2 \min _{\xi \in \mathcal{C}^{\prime}}\left(y_{\mathrm{qc}}\right)_{\xi}^{\prime}\right)$. Hence, if we require that (5.7) holds with $\delta_{1}=$ $c_{\mathrm{qc}}\left(y_{\mathrm{qc}}\right) /\left(8 \bar{C}_{3}\right)$, then we obtain

$$
\left\|D \mathscr{F}(0)^{-1}\right\|_{L\left(\mathscr{U}^{-1,2}, \mathscr{U}^{1,2}\right)} \leq 1 / c_{\mathrm{a}}\left(y_{\mathrm{qc}}\right) \leq \sigma \leq 2 / c_{\mathrm{qc}}\left(y_{\mathrm{qc}}\right) .
$$


3. Lipschitz bound: The proof of a Lipschitz bound for $D \mathscr{F}$ is a verbatim repetition of step 3 in the proof of Theorem [5.1. For some fixed $0<\delta<1$, we obtain that, if $\left\|w_{i}^{\prime}\right\|_{\ell_{\varepsilon}^{2}} \leq 2 \eta \sigma, i=1,2$, then

$$
\left\|D \mathscr{F}\left(w_{1}\right)-D \mathscr{F}\left(w_{2}\right)\right\|_{L\left(\mathscr{U}^{1,2}, \mathscr{U}^{-1,2}\right)} \leq \varepsilon^{-1 / 2} L^{\prime}\left\|w_{1}-w_{2}\right\|_{\mathscr{U}^{1,2}},
$$

where $L^{\prime}$ depends only on $C_{3}\left(\delta \min _{\xi}\left(y_{\mathrm{qc}}\right)_{\xi}^{\prime}\right)$. The actual Lipschitz constant is again set to $L=\varepsilon^{-1 / 2} L^{\prime}$.

4. Conclusion: The conclusion of the proof follows precisely step 4 in the proof of Theorem 5.1. The different constant in the error estimate is due to the sharper definition of $\sigma$.

Remark 5.4. Theorem 5.3 is the first rigorous a posteriori error estimate of this kind for the QNL-QC method. However, the basic idea behind the result, that is, to use a posteriori information about the approximate solution to deduce the existence of an exact solution, is not new. A similar approach to the one used in the proof of Theorem 5.3 can be found in the work of Plum [23] on numerical enclosure methods for semilinear differential equations. A detailed analysis of these ideas, in the context of a posteriori error control for finite element methods was given in [20]. The first application of this idea in an analysis of a QC method was presented in [21, Thm. 5.1]. The latter result differs from Theorem 5.3 not only in the type of QC method analyzed, but also in the choice $\mathscr{U}^{1, \infty}$ for the function space settings. While that choice gives uniform neighbourhoods and thus asymptotically sharper existence conditions, a sharp stability result such as Theorem 4.7 would be difficult to prove. Moreover, a generalization of [21, Thm. 5.1] to higher dimensions seems virtually impossible.

\section{CONCLUSION}

In order to outline a new framework for the analysis of $\mathrm{QC}$ methods, we have chosen a particularly simple setting, admitting only second-neighbour pair interactions, and disregarding finite element coarse graining of the continuum region. Extensions of this work to more complex interaction models and to coarse-grained models can be found in [13, 22. The most interesting extension, however, will be to the application-relevant two- and three-dimensional settings. While great care was taken in this paper that a clear path for such a generalization is available, many interesting questions will need to be carefully considered in order to establish the consistency and (linear) stability of the QC method. Even the relatively basic question of consistency is still open for a general finite range interaction model, however, the work in [10], for flat interfaces, provides a promising starting point. Moreover, in a recent paper Shapeev [25] constructs an energy-based coupling scheme for pair potentials in two dimensions that is consistent for general interface geometries. No results on the stability of the QC method in $2 \mathrm{D} / 3 \mathrm{D}$ can be found in the literature at present.

\section{ACKNOWLEDGEMENTS}

The author is greatful for helpful discussions with M. Dobson, X. H. Li, and M. Luskin, and their comments on a previous draft of the paper. 


\section{REFERENCES}

1. M. Arndt and M. Luskin, Error estimation and atomistic-continuum adaptivity for the quasicontinuum approximation of a Frenkel-Kontorova model, Multiscale Model. Simul. 7 (2008), 147-170. MR.2399541 (2009a:65393)

2. _ Goal-oriented adaptive mesh refinement for the quasicontinuum approximation of a Frenkel-Kontorova model, Computer Methods in Applied Mechanics and Engineering 197 (2008), 4298-4306. MR2463663 (2009j:74029)

3. X. Blanc, C. Le Bris, and F. Legoll, Analysis of a prototypical multiscale method coupling atomistic and continuum mechanics, M2AN Math. Model. Numer. Anal. 39 (2005), no. 4, 797-826. MR2165680 (2006i:74023)

4. M. Dobson and M. Luskin, Analysis of a force-based quasicontinuum approximation, M2AN Math. Model. Numer. Anal. 42 (2008), no. 1, 113-139. MR2387424 (2009f:74003)

5. _ An optimal order error analysis of the one-dimensional quasicontinuum approximation, SIAM J. Numer. Anal. 47 (2009), no. 4, 2455-2475. MR.2525607

6. M. Dobson, M. Luskin, and C. Ortner, Accuracy of quasicontinuum approximations near instabilities, J. Mech. Phys. Solids 58 (2010), 1741-1757.

7. __ Iterative methods for the force-based quasicontinuum approximation, Comput. Mathods Appl. Mech. Engrg., doi: 10.1016/j.cma.2010.07.008, 2010.

8. Sharp stability estimates for the force-based quasicontinuum approximation of homogeneous tensile deformation, Multiscale Model. Simul. 8 (2010), no. 3, 782-802.

9. Stability, instability, and error of the force-based quasicontinuum approximation, Arch. Ration. Mech. Anal. 197 (2010), no. 1, 179.

10. W. E, J. Lu, and J.Z. Yang, Uniform accuracy of the quasicontinuum method, Phys. Rev. B 74 (2004), no. 21, 214115

11. W. E and P. Ming, Cauchy-Born rule and the stability of crystalline solids: dynamic problems, Acta Mathematicae Applicatae Sinica, English Series 23 (2007), no. 4, 529-550. MR2329067 (2008d:35132)

12. - Cauchy-Born rule and the stability of crystalline solids: static problems, Arch. Ration. Mech. Anal. 183 (2007), no. 2, 241-297. MR2278407 (2009b:74006)

13. X.H. Li and M. Luskin, A generalized quasi-nonlocal atomistic-to-continuum coupling method with finite range interaction, arXiv:1007.2336v1

14. P. Lin, Convergence analysis of a quasi-continuum approximation for a two-dimensional material without defects, SIAM J. Numer. Anal. 45 (2007), no. 1, 313-332. MR2285857 (2008f:74006)

15. M. Luskin and C. Ortner, An analysis of node-based cluster summation rules in the quasicontinuum method, SIAM J. Numer. Anal. 47 (2009), no. 4, 3070-3086. MR2551158

16. R.E. Miller and E.B. Tadmor, Benchmarking multiscale methods, Modelling and Simulation in Materials Science and Engineering 17 (2009), p. 053001.

17. - The Quasicontinuum Method: Overview, Applications and Current Directions, Journal of Computer-Aided Materials Design 9 (2003), 203-239.

18. P. Ming and J.Z. Yang, Analysis of a one-dimensional nonlocal quasi-continuum method, Multiscale Model. Simul. 7 (2009), no. 4, 1838-1875. MR.2539201(2010g:74073)

19. M. Ortiz, R. Phillips, and E.B. Tadmor, Quasicontinuum Analysis of Defects in Solids, Philosophical Magazine A 73 (1996), no. 6, 1529-1563.

20. C. Ortner, A posteriori existence in numerical computations, SIAM J. Numer. Anal. 47 (2009), no. 4, 2550-2577. MR2525611 (2010g:65189)

21. C. Ortner and E. Süli, Analysis of a quasicontinuum method in one dimension, M2AN Math. Model. Numer. Anal. 42 (2008), no. 1, 57-91. MR2387422(2009e:74006)

22. C. Ortner and H. Wang, A priori error analysis for energy-based quasicontinuum approximations of a periodic chain, OxMOS preprint no. 30/2010, Mathematical Institute, University of Oxford.

23. M. Plum, Computer-assisted enclosure methods for elliptic differential equations, Linear Algebra Appl. 324 (2001), no. 1-3, 147-187, Special issue on linear algebra in self-validating methods. MR 1810529 (2002a:65080)

24. S. Prudhomme, P.T. Bauman, and J.T. Oden, Error control for molecular statics problems, International Journal for Multiscale Computational Engineering 4 (2006), no. 5-6, 647-662. 
25. A. Shapeev, Consistent energy-based atomistic/continuum coupling for two-body potential: 1d and 2d case, arXiv:1010.0512

26. V.B. Shenoy, R. Miller, E.B. Tadmor, D. Rodney, R. Phillips, and M. Ortiz, An adaptive finite element approach to atomic-scale mechanics-the quasicontinuum method, J. Mech. Phys. Solids 47 (1999), no. 3, 611-642. MR.1675219 (99k:73116)

27. T. Shimokawa, J.J. Mortensen, J. Schiotz, and K.W. Jacobsen, Matching conditions in the quasicontinuum method: Removal of the error introduced at the interface between the coarsegrained and fully atomistic region, Phys. Rev. B 69 (2004), no. 21, 214104.

Mathematical Institute, 24-29 St Giles', Oxford OX1 3LB, United Kingdom

E-mail address: ortner@maths.ox.ac.uk 